\title{
One-year outcomes of a therapist-supported, smartphone-based intervention for elevated symptoms of depression and anxiety
}

Marcos Economides, PhD ${ }^{a^{*},}$, Kristian Ranta, MSc ${ }^{a}$, Albert Nazander, MSc a, Outi Hilgert, MD a, Philippe R Goldin, PhD ${ }^{b}$, Anu Raevuori, MD PhD a,c,d, Valerie Forman-Hoffman, PhD, MPH a

\footnotetext{
a Meru Health Inc.

b Betty Irene Moore School of Nursing, University of California Davis, Sacramento, CA, United States

c Department of Adolescent Psychiatry, Helsinki University Central Hospital, Helsinki, Finland

d Clinicum, Department of Public Health, University of Helsinki, Helsinki, Finland

*corresponding author: marcos@meruhealth.com
}

\begin{abstract}
Depression is common and severely impacts physical, psychological and social functioning. To address access barriers to care, we developed Ascend - a smartphone-delivered, therapistsupported, 8-week intervention based on several evidence-based psychological treatments for depression and anxiety. We examined whether the previously-reported, end-of-treatment improvements among 102 adults with elevated symptoms of depression extended up to 12 months post-treatment for depression symptoms (measured by the Patient Health Questionnaire [PHQ-9]), and up to 6 months post-treatment for anxiety symptoms (added to the intervention later, and measured by the Generalized Anxiety Disorder scale [GAD-7]). An intention-to-treat analysis showed that participants maintained clinically significant improvements in depression (mean PHQ-9 reduction=6.67, Hedges' $g=1.14$ [0.78 to 1.49$]$ ) and in anxiety (mean GAD-7 reduction=4.26, Hedges' $g=0.91$ [0.54 to 1.28 ]) at follow-up. Also, $60 \%$ of participants above the cutoff for major depression at baseline (PHQ $\geq 10$ ) experienced $\geq 50 \%$ reduction in PHQ-9 score and had PHQ-9 $<10$ at follow-up. Future randomized trials are warranted to test Ascend as a scalable solution to the treatment of depression and/or symptoms of anxiety.
\end{abstract}

Keywords: digital health, smartphone intervention, depression, anxiety, online therapy, mHealth 


\section{Introduction}

Depression is a common mental health disorder and one of the leading causes of disease burden and disability worldwide ${ }^{1-3}$. Individuals with depression have a reduced capacity to work and function in daily life, causing major economic and societal costs ${ }^{4-6}$. By 2030, depression is predicted to pose the largest burden of disease in high-income countries, surpassing heart disease, dementia, and alcoholrelated disorders ${ }^{1}$.

Although there are several effective pharmacological ${ }^{7}$ and psychological ${ }^{8,9}$ treatments for depression, less than half of all individuals who require treatment actually receive it ${ }^{10,11}$. Barriers to treatment include financial and time constraints, long wait periods, a shortage of trained professionals, and fear of stigmatization ${ }^{12,13}$. When treated with antidepressants an estimated 30 $50 \%$ of patients do not experience significant symptom reduction ${ }^{14,15}$, up to $86 \%$ of patients report mild to severe side-effects ${ }^{16,17}$, and only a minority of patients maintain a state of long-term remission ${ }^{18}$. Estimates suggest that up to $70 \%$ of individuals with depressive disorders have a comorbid anxiety disorder, which renders treatment even more challenging ${ }^{19}$. Further, at least $75 \%$ of patients who are referred to in-person psychotherapy never enter treatment ${ }^{20,21}$. Thus, there is an urgent need for novel, evidence-based treatments for depression and anxiety that overcome these barriers.

Recently, online-based psychological interventions have been developed as a means to address this need ${ }^{22}$. Online interventions offer several advantages over traditional treatment modalities, including i) large-scale accessibility and scalability, ii) low costs, iii) patient anonymity and privacy, iv) standardized content that is less dependent on therapist skills, and v) flexible usage at a selfdetermined time and pace, which is thought to enhance self-efficacy ${ }^{23}$. Several meta-analyses have shown that online interventions for depression and anxiety that utilize cognitive behavioral therapy (CBT) ${ }^{24-27}$ or mindfulness meditation (MM) ${ }^{28}$ are effective at alleviating symptoms with small-tomoderate effect sizes. Further, preliminary evidence suggests that online CBT can be used by most individuals with symptoms of depression, regardless of their socioeconomic background or symptom severity ${ }^{29}$, and is equally effective when compared to CBT that is delivered in-person ${ }^{30}$.

In recent years, online-based interventions for mental health have been extended to the domain of smartphone applications, or "apps". Smartphone ownership has seen rapid worldwide growth ${ }^{31}$, 
and survey data suggests that smartphone-based interventions (SBIs) may be preferred over other online formats by health care consumers ${ }^{32,33}$. The advantages of SBIs include i) portability and flexibility of use, ii) the monitoring of activity, symptoms and progression in real-time, iii) the provision of personalized feedback, motivational support and targeted care, and iv) the potential to improve adherence to treatment ${ }^{34}$. Preliminary evidence suggests that SBIs are a promising means to treat depression and anxiety, with recent meta-analyses reporting small-to-moderate reductions in clinical symptoms across 27 total studies ${ }^{35,36}$. However, few authors report on outcomes of commercially-developed SBIs for mental health, making it difficult to evaluate their utility ${ }^{34,37}$. Further, there is a dearth of evidence regarding whether the effects of SBIs and other online interventions for symptoms of depression and anxiety are maintained post-treatment ${ }^{25}$.

We recently evaluated the feasibility of the Meru Health Ascend intervention, a novel, 8-week SBI for elevated symptoms of depression and anxiety that's assisted by a remote therapist ${ }^{38}$. The intervention was found to be feasible and was associated with a post-intervention reduction in depression. In the present study, we extend these findings by investigating whether Ascend is also associated with a reduction in anxiety, which was added to the intervention as a treatment outcome at a later date. Critically, we also investigated whether reductions in depression and anxiety are maintained at 1-year and 6-month follow-up, respectively, lending important insights as to the realworld, long-term impact of an SBI for the treatment of common mental health problems. We hypothesized that Ascend would be associated with a reduction in anxiety symptoms at the end of treatment, and that post-intervention reductions in symptoms of depression and anxiety would be maintained at 1-year and 6-month follow-up assessments, respectively.

\section{Methods}

\section{Research Design}

We used a quasi-experimental research design that included a single-arm pre- and post-intervention assessment of outcomes. Symptoms of depression were measured pre-intervention ("baseline"), at the end of the 8-week intervention, and at 1, 3, 6, and 12 months post-intervention. Symptoms of anxiety were measured at baseline, at week- 4 of the intervention, at the end of the 8-week intervention, and at 1,3 , and 6 months post-intervention.

\section{Participants}


The present study included adult patients treated at the Meru Health Online Clinic, a national remote healthcare provider that currently operates in the US and Finland. The clinic has had a rolling enrolment since March 2017. At the time of this study, 197 enrollees had passed the 6-month postintervention outcome window (recruited between March 2017 and June 2018), whilst 102 had passed the 12-month post-intervention outcome window (recruited between March and December 2017). Our primary analysis includes the latter group, although we also report PHQ-9 results from the former group with 6-month post-intervention outcomes only. In addition, since anxiety measures were added to the intervention in December 2017, 102 of the 197 enrollees with 6-month post-intervention outcomes also had anxiety data, whilst 12-month post-intervention anxiety data was not yet available.

Participants were recruited via online Facebook adverts that sought participants for an SBI for depression that included self-guided smartphone-delivered content, private access to a therapist via messaging, and an anonymous weekly group chat with other participants. Participants were given free access to the app and trained on how to use the weekly group chat feature, as well as how to communicate with their assigned therapist, prior to beginning the intervention. Participant demographics (age, gender, and antidepressant medication status) were acquired at the start of the intervention via an intake questionnaire administered online. Since medication status was introduced in July 2017, this data is absent for the first 33 participants. Outcome measures were administered via the app for all within-intervention time-points (including immediately postintervention), and via email for all follow-up time-points.

For inclusion, participants had to provide informed consent via the Meru Health app, own a smartphone, have at least mild symptoms of depression (a score $\geq 5$ on the Patient Health Questionnaire [PHQ-9] at baseline), and acknowledge/demonstrate the ability to commit to a minimum of 20 minutes of practice per day, for 6 days per week, across the 8-week intervention (as judged by both the participant and their assigned therapist). Exclusion criteria included a previous suicide attempt, severe active suicidal ideation with a specific plan, severe self-harm, active substance abuse, and a history of psychosis. Inclusion/exclusion criteria were assessed prior to enrolment via phone interviews between study participants and intervention therapists, as per the standard treatment procedure at the Meru Health Online Clinic. Participants were not compensated for their time but could participate in the intervention for free. Institutional review board exemption was granted by Pearl IRB for analyses of previously collected and de-identified data. 
An a priori sample size calculation was performed for comparing patient-reported outcome measures (PHQ-9 and GAD-7 scores) at baseline and follow-up time-points. Using an alpha level of .05 , a power of 0.8 , and a medium effect size of $0.5,33$ subjects were needed. Thus, the present study was sufficiently powered to detect a medium effect, even after accounting for substantial dropout over the course of the 1-year follow-up period.

\section{Intervention}

The Meru Health Ascend intervention has been described in detail previously ${ }^{38}$. Briefly, the intervention consists of 8 modules delivered sequentially over an 8-week period, that include content derived from evidence-based practices such as Mindfulness-Based Stress Reduction (MBSR $\left.{ }^{39}\right)$, Mindfulness-Based Cognitive Therapy $\left(\mathrm{MBCT}^{40}\right)$, Cognitive-Behavioral Therapy $\left(\mathrm{CBT}^{41}\right)$, and Behavioral Activation Therapy (BAT ${ }^{42}$ ). The content includes text, video, audio-guided mindfulness meditation exercises, infographics that illustrate CBT principles, and journal prompts. Daily content and practices range from 10-30 minutes, except for the first day of each week, in which a series of introductory videos extend the content to a maximum of 45 minutes.

A licensed therapist (employed by Meru Health) provides support to participants via messaging (and less frequently, phone calls) as needed, and reviews practice logs using a provider "dashboard" and electronic medical records that detail individual progress (including participant engagement and patient-reported outcomes to date). In total, therapists allocate approximately 20 minutes (on average) per week per participant. Interaction between therapist and participant can be initiated via either party. Therapists are instructed to conduct a phone-based assessment for any participants that show signs of mental deterioration during and/or immediately after the intervention. In case of an emergency, such as severe suicidality, the intervention includes a written security plan which all participants are required to review with their therapist prior to engaging with the intervention.

Participants are enrolled in groups of between 10-15 individuals that work through the intervention at the same time, and can provide anonymous support to one another via a discussion board within the app. Specifically, participants can post anonymous reflections on practices and lessons to the discussion board, to which their therapist can respond freely, and to which other group members can respond with pre-written empathy statements and/or emoticons. Free cross-talk between participants is not allowed. 
In Finland, Meru Health is approved by the Finnish National Supervisory Authority for Welfare and Health (Valvira approval number V/25535/2017) and is compliant with the EU General Data Protection Regulation (GDPR). In the USA, Meru Health is compliant with HIPAA legislation. All protected health information (PHI) is kept in a HIPAA-compliant electronic medical record, which is housed in cloud-based storage hosted by a company named Datica (https://datica.com/). All data is encrypted in transit, end-to-end, and at rest.

\section{Measures}

Patient Health Questionnaire. The PHQ-9 is a 9-item depression scale, derived from the full PHQ, and is one of the most widely used instruments to screen for the presence and severity of depression in primary care ${ }^{43}$. Participants rate each item on a Likert scale from 0 (not at all) to 3 (nearly every day), with total scores ranging from 0 to 27. In general, a score of 10 or above suggests the presence of major depression, and scores of 5, 10, 15, and 20 are taken as cut-off points for mild, moderate, moderately severe, and severe depression, respectively. The PHQ-9 has excellent internal consistency (Cronbach's $\alpha$ of 0.89 in primary care settings), and excellent test-retest reliability ${ }^{44}$. In their original validation study, Kroenke and colleagues defined a clinically significant improvement in depression as a 50\% reduction in PHQ-9 score combined with a post-intervention score $<10$ (for participants with baseline scores $\geq 10$ ), and this definition has been further validated in a comparison study ${ }^{45}$. A similar yet more liberal definition for clinically significant change was also proposed by Löwe and colleagues as a PHQ-9 score reduction of $\geq 5^{46}$.

Generalized Anxiety Questionnaire. The GAD-7 is a 7-item scale, used extensively in outpatient and primary care settings to screen for the presence and severity of an anxiety disorder ${ }^{47}$. Participants rate each item on a Likert scale from 0 (not at all) to 3 (nearly every day), with total scores ranging from 0 to 21 . In general, a score of 10 or above is suggestive of the presence of anxiety to the extent that further evaluation is warranted, and scores of 5, 10, and 15 are taken as cut-off points for mild, moderate, and severe anxiety, respectively. The GAD-7 has excellent reliability and internal consistency (Cronbach's $\alpha$ of 0.89 ) and has been validated in both the general population and primary care settings 47,48 . A clinically significant improvement in anxiety symptoms has previously been defined as a GAD-7 score reduction of $\geq 3^{49}$.

\section{Statistical analysis}


Descriptive statistics were calculated for participant demographics (see Table 1) and week-by-week engagement metrics (see Table 2). We used logistic regression to explore whether baseline characteristics were predictive of the completion of outcome measures at 6 - and 12-months postintervention. Explanatory variables included age, gender, intervention engagement (total days in which participants engaged with >3 minutes of app-based meditation), and baseline PHQ-9 or GAD-7 scores.

Outcome measures were analyzed using an intention-to-treat (ITT) analysis in which all participants with outcome measures at baseline were included, regardless of intervention engagement or attrition. We used linear mixed effects models (LMMs) implemented through LME4 ${ }^{50}$, and Tukey contrasts to compare between time-points (using the "multcomp" package ${ }^{51}$ ) in the statistical computing software R (version 3.5.2, The R Project for Statistical Computing). LMMs are capable of handling missing data and are considered superior to other ITT approaches such as last observation carried forward (LOCF) ${ }^{52}$. We modelled a single within-subject factor 'time' (as a fixed effect) and a separate baseline for each participant (random-intercept model). Time was modelled as a categorical predictor, and we therefore did not enforce a linear relationship between time and outcome measures. We report the contrast estimate, $95 \% \mathrm{Cl}$ on the estimate, and $p$ value. $p$ values $<.05$ were considered significant. The estimated marginal mean (EMM) and standard error for each time-point was calculated using the "emmeans" package in R.

Since LMMs rely on data being 'missing at random', an assumption that's difficult to verify in clinical research, we also implemented an LMM-based pattern-mixture model (PMM) for the analysis of PHQ-9 (depression) scores. PMMs are based on a joint modelling of outcomes and missingness and can account for data that is 'missing not at random' ${ }^{53}$. We modified the EMM from the previously described LMM at the 6- and 12-month time-points based on specific clinical assumptions about participants with missing data. We identified 3 patterns of data: 1) participants with complete outcome data at baseline and 12-month follow-up $(n=52) ; 2)$ participants who were lost to followup during or immediately post-intervention ( $n=21)$; and, 3) participants who were lost at the $1,3,6$, or 12-month follow-up time-points $(n=29)$. We used the conservative approach of assuming that participants belonging to pattern 2 did not benefit from the intervention and set the EMM at 6 - and 12-months follow-up for these participants equal to the EMM at baseline. For group 3, we estimated the EMM at 6-and 12-months follow-up as being equal to the EMMs across the first 3 time-points of the intervention (baseline to week-4). The overall treatment effect at 6- or 12-months follow-up was then defined as a linear combination of EMMs for the 3 different patterns, weighted by the 
proportion of participants belonging to each pattern. The standard error for this estimate was calculated using the delta method (via the "car" package in $\mathrm{R}^{54}$ ), and a test of the null hypothesis of no treatment effect was performing using a Wald statistic. For a full description of the LMM-based PMM procedure see Ratitch and O'Kelly ${ }^{55}$.

For comparisons with previous literature, we calculated effect size (Hedges' $g$ ) immediately postintervention, and at 6-and 12-months after the intervention, as the difference in outcome scores from baseline divided by the pooled weighted standard deviation (based on observed outcome scores only). For the PMM, effect size (Cohen's $d$ ) was calculated as the estimated reduction in outcome score (from the LMM-based PMM) divided by the standard deviation of observed outcome scores at baseline. We also calculated the percentage of participants who met the definition for clinically significant improvement in PHQ-9 symptoms post-intervention and at 12-month follow-up, defined as either a 5-point reduction in PHQ-9 score from baseline, or a 50\% reduction in combination with a post-intervention score $<10$ (for participants with baseline scores $\geq 10$ ).

Lastly, we performed exploratory multiple regressions to test whether participant demographics and engagement metrics predicted outcome change from baseline to 6 - and 12 -months postintervention.

\section{Results}

\section{Demographics and participation}

Participant demographics are presented in Table 1. On average participants were 33 years of age, had baseline symptoms above the cutoff for major depression (mean PHQ-9 = 12.8), and were predominantly female. 80 participants were based in Finland whilst 22 were based in the US. Approximately one third of participants were taking antidepressant medication at the start of the intervention. 20 participants (19.6\%) dropped out of the intervention, where dropout was defined as less than 4 weeks of active participation during the 8-week intervention, combined with incomplete PHQ-9 (depression) scores immediately post-intervention.

Just over half (51\%) of all participants completed post-intervention follow-up outcomes (see Table 1). Logistic regression revealed that participants with higher depression scores at baseline $(B=-0.11$, $p=0.019)$, and those who engaged with the intervention on a higher volume of days $(B=-0.05, p=$ 
0.009), were more likely to complete the PHQ-9 at 12-months post-intervention. Intervention engagement also positively predicted completion of GAD-7 at 6 -months post-intervention $(B=-0.07$, $p=<0.001)$, but baseline GAD-7 scores did not $(p<0.05)$.

\begin{tabular}{|c|c|c|c|c|c|c|c|}
\hline & $\begin{array}{c}\text { All } \\
\text { participants }\end{array}$ & $\begin{array}{l}\text { Completed } \\
\text { 8-week } \\
\text { outcomes }\end{array}$ & $\begin{array}{l}\text { Completed } \\
6 \text {-month } \\
\text { outcomes }\end{array}$ & $\begin{array}{l}\text { Completed } \\
12 \text {-month } \\
\text { outcomes }\end{array}$ & $\begin{array}{c}\text { Did not } \\
\text { complete } \\
\text { 8-week } \\
\text { outcomes }\end{array}$ & $\begin{array}{c}\text { Did not } \\
\text { complete } \\
6 \text {-month } \\
\text { outcomes }\end{array}$ & $\begin{array}{c}\text { Did not } \\
\text { complete } \\
\text { 12-month } \\
\text { outcomes }\end{array}$ \\
\hline n (\%) & $102(100)$ & 83 (81.4) & 44 (43.1) & $52(51)$ & 19 (18.6) & 58 (56.9) & $50(49)$ \\
\hline $\begin{array}{l}\text { Age in years, } \\
\text { mean (SD) }\end{array}$ & $32.9(10.3)$ & $33.2(10.4)$ & $33.5(11.0)$ & $31.3(11.0)$ & $31.9(10.0)$ & $32.5(9.9)$ & $34.6(9.4)$ \\
\hline \multicolumn{8}{|l|}{ Gender } \\
\hline Male, n (\%) & $23(22.5)$ & 19 (22.9) & $11(25)$ & $10(19.2)$ & $4(21.0)$ & $12(20.7)$ & $13(26)$ \\
\hline Female, $n(\%)$ & $79(77.5)$ & $64(77.1)$ & $33(75)$ & $42(80.8)$ & $15(79.0)$ & $46(79.3)$ & $37(74)$ \\
\hline \multicolumn{8}{|l|}{ Antidepressants } \\
\hline Yes, n (\%) & $25(24.5)$ & $18(21.7)$ & $7(15.9)$ & $7(13.5)$ & $7(36.8)$ & $18(31.0)$ & $18(36)$ \\
\hline No, n (\%) & $44(43.1)$ & $37(44.6)$ & $24(54.6)$ & $22(42.3)$ & $7(36.8)$ & $20(34.5)$ & $22(44)$ \\
\hline Unknown, n (\%) & $33(32.4)$ & $28(33.7)$ & $13(29.6)$ & $23(44.2)$ & $5(26.3)$ & $20(34.5)$ & $10(20)$ \\
\hline \multicolumn{8}{|l|}{ Country } \\
\hline Finland, $\mathrm{n}(\%)$ & $80(78.4)$ & $67(80.7)$ & $36(81.8)$ & $48(92.0)$ & $13(68.0)$ & 44 (75.9) & $32(64.0)$ \\
\hline USA, n (\%) & $22(21.6)$ & $16(19.3)$ & $8(18.2)$ & $4(8.0)$ & $6(32.0)$ & $14(24.1)$ & $18(36.0)$ \\
\hline $\begin{array}{l}\text { Baseline PHQ-9 } \\
\text { score, mean (SD) * }\end{array}$ & $12.8(5.2)$ & $13.0(5.4)$ & $13.2(5.8)$ & $14.3(5.0)$ & $11.6(4.6)$ & $12.5(4.8)$ & $11.2(5.0)$ \\
\hline
\end{tabular}

Table 1. Participant demographics and relevant baseline data. * Significantly associated with the presence of PHQ-9 scores at 12-month follow-up.

\section{Engagement}

Week-by-week intervention engagement rates are summarized in Table 2. On average participants engaged with the intervention on 31.3 days $(S D=13.5, \min =3, \max =56)$, which corresponds to $55.9 \%$ of intervention days (where engagement is defined as $>3$ mins of app-based mindfulness meditation on a given day). Participants completed an average of 9.79 hours of mindfulness-based exercises $(S D=5.01, \min =0.79, \max =24.7)$, and had contact with their therapist on 13.1 days $(S D=$ $8.34, \min =0, \max =35)$, or $23.4 \%$ of intervention days. 68 participants $(66.7 \%)$ completed at least one app-based meditation practice on each of the 8 weeks of the intervention. As reported in a previous publication ${ }^{38}$, the mean number of days of intervention engagement $\left(F_{7,707}=42.4, p<\right.$ $0.001)$ and the mean number of days of therapist contact $\left(F_{7,707}=5.37, p<0.001\right)$ decreased from week 1 to week 8 (see Table 2). 
Out of 102 participants, $81.4 \%$ completed the PHQ-9 immediately post-intervention, whilst $43.1 \%$ and $51 \%$ completed the PHQ-9 at 6 - and 12 -months post-intervention, respectively.

\begin{tabular}{|l|c|c|c|c|c|c|c|c|}
\cline { 2 - 9 } \multicolumn{1}{c|}{} & \multicolumn{10}{c|}{ Week } \\
\hline Metric & 1 & 2 & 3 & 4 & 5 & 6 & 7 & 8 \\
\hline Active days, mean (SD) & 5.02 & 4.65 & 4.54 & 4.13 & 3.76 & 3.59 & 2.95 & 2.67 \\
& $(1.81)$ & $(1.89)$ & $(1.95)$ & $(1.95)$ & $(2.19)$ & $(2.34)$ & $(2.21)$ & $(2.20)$ \\
\hline Meditation minutes, & 93.9 & 105.9 & 80.6 & 76.1 & 82.6 & 67.7 & 39.0 & 41.5 \\
mean (SD) & $(39.0)$ & $(52.6)$ & $(40.0)$ & $(47.6)$ & $(60.5)$ & $(55.2)$ & $(39.8)$ & $(39.5)$ \\
\hline Days with therapist & 2.02 & 1.90 & 1.65 & 1.75 & 1.47 & 1.46 & 1.43 & 1.42 \\
contact, mean (SD) & $(1.27)$ & $(1.53)$ & $(1.40)$ & $(1.40)$ & $(1.42)$ & $(1.45)$ & $(1.43)$ & $(1.37)$ \\
\hline
\end{tabular}

Table 2. Week-by-week intervention engagement across all participants. 'Active days' corresponds to $>3$ minutes of app-based mindfulness practice on a given day.

\section{Depression symptoms}

Participants reported clinically significant improvements in depression symptoms (PHQ-9 scores) from baseline to post-intervention ( 5.50 reduction, $95 \% \mathrm{Cl} 4.58$ to $6.42, \mathrm{p}<0.001$, Hedges' $g=1.02$ [0.71 to 1.32]; Figure $1 \mathrm{~A}$ ). This improvement was maintained at 6 -months ( 6.76 reduction, $95 \% \mathrm{Cl}$ 5.61 to $7.90, \mathrm{P}<0.001$, Hedges' $g=1.30$ [0.91 to 1.68$]$ ) and 12-months (6.67 reduction, $95 \% \mathrm{Cl} 5.59$ to $7.75, p<0.001$, Hedges' $g=1.14$ [0.78 to 1.49 ]) post-intervention. The improvement at 6 -months remained robust when including an additional 95 participants (total $n=197$ ) with outcomes available at 6 -months post-intervention only $(6.53$ reduction, $95 \% \mathrm{Cl} 5.73$ to $7.33, \mathrm{p}<0.001$, Hedges' $g=1.28[1.00$ to 1.55$])$. 

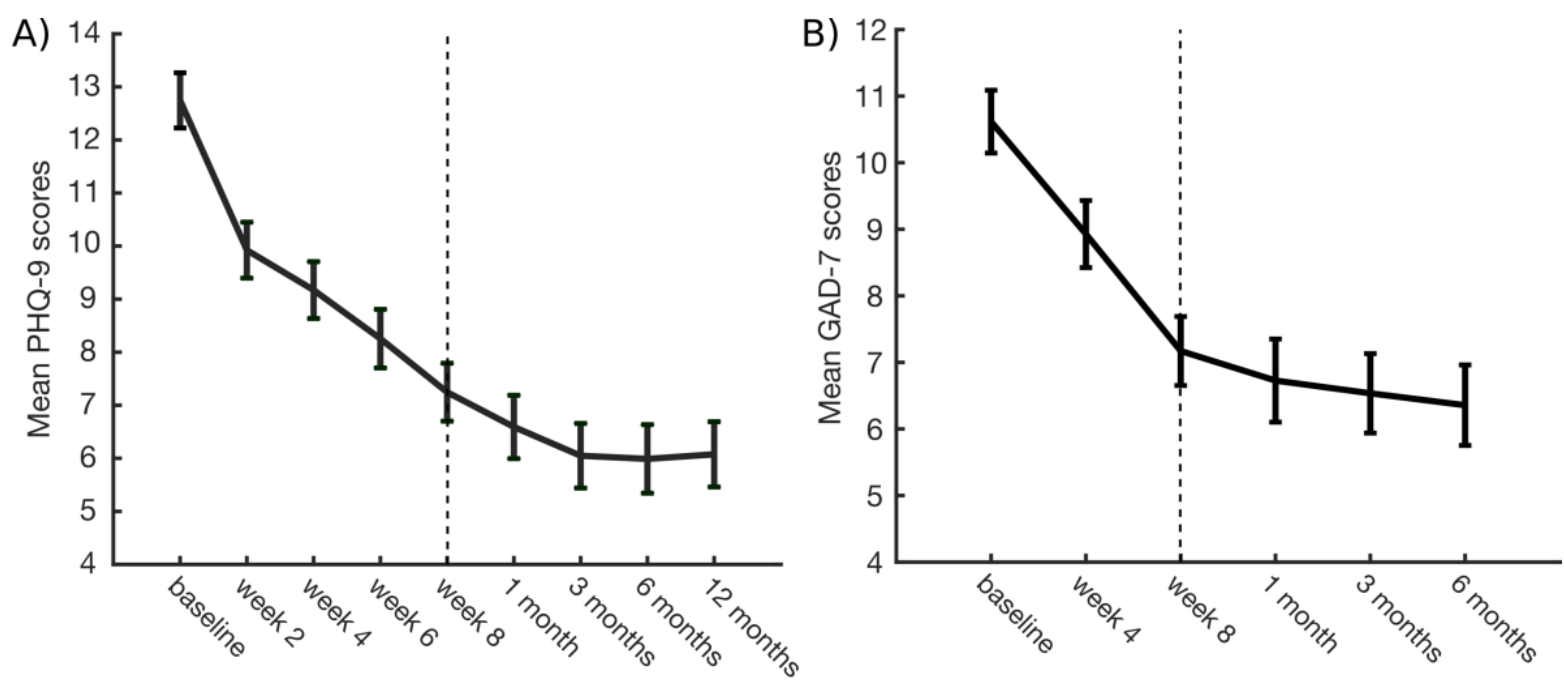

Figure 1. Estimated marginal means for PHQ-9 (A) and GAD-7 (B) scores across all available timepoints. The dotted line indicates the last week of the 8-week intervention. Error bars represent standard error of the mean (SEM).

When considering participants with PHQ-9 scores $\geq 10$ at baseline $(n=83), 48 \%$ reported a clinically significant improvement immediately post-intervention, which increased to $60 \%$ at 12 -month followup (defined as post-intervention score $<10$ combined with $\geq 50 \%$ symptom reduction). When including all participants and using the more liberal definition of a 5-point reduction in PHQ-9 symptoms, $56 \%$ reported clinically significant improvements immediately post-intervention, which increased to $77 \%$ at 12 -month follow-up.

We also estimated PHQ-9 scores at 6- and 12-months post-intervention under the conservative assumption that participants with missing data did not experience any long-term benefit from the intervention, or benefitted only marginally (see Methods). This approach revealed more modest yet significant reductions in PHQ-9 scores relative to baseline at both 6-months (4.35 reduction, $95 \% \mathrm{Cl}$ 3.65 to $5.06, \mathrm{P}<0.001$, Cohen's $d=0.83$ [0.43 to 1.24 ]) and 12 -months ( 4.31 reduction, $95 \% \mathrm{Cl} 3.63$ to $4.99, \mathrm{p}<0.001$, Cohen's $d=0.82$ [0.42 to 1.23$]$ ]) post-intervention.

\section{Anxiety symptoms}

Participants reported clinically significant improvements in anxiety (GAD-7 scores) from baseline to post-intervention ( 3.45 reduction, $95 \% \mathrm{Cl} 2.51$ to $4.38, p<0.001$, Hedges' $g=0.69$ [0.38 to 1.00]; Figure $1 \mathrm{~B})$. This improvement was maintained at 6 -months post-intervention $(4.26$ reduction, $95 \% \mathrm{Cl}$ 3.14 to $5.38, \mathrm{P}<0.001$, Hedges' $g=0.91$ [0.54 to 1.28$]$ ). 


\section{Predictors of outcome change}

None of the reported engagement metrics or participant demographics were predictive of score change from baseline at 6- and 12-month follow-up, for either PHQ-9 or GAD-7 (all p > 0.05).

\section{Discussion}

\section{Principal findings}

The Meru Health Ascend intervention is a newly-developed, smartphone-based, therapist-supported intervention for depression and anxiety designed to overcome common barriers to treatment. A recent feasibility study reported that the intervention is feasible and associated with reduced depression symptoms immediately after the intervention ${ }^{38}$. The present follow-up study extends these findings by demonstrating that the intervention is associated with clinically significant reductions in symptoms of both depression and anxiety, and that these reductions are maintained up to 1 year and 6 months after intervention completion, respectively.

Our analysis revealed a larger effect size for the reduction in depression symptoms 12 -months postintervention $(g=1.14)$ than was reported by a recent meta-analysis comparing smartphone-based interventions for depression to inactive control groups ${ }^{35}(g=0.56)$. However, the present study was uncontrolled, and the large effect reported here is consistent with other within-group (uncontrolled) effect sizes for app- and online-based depression interventions ${ }^{56-58}$. The observed reduction in depression symptoms remained significant when making the conservative assumption that participants with missing data did not experience any long-term benefit from the intervention. However, the average reduction in depression under this assumption was marginally below the threshold for clinical significance (5-point change in PHQ-9 symptoms). We also found medium-tolarge effects for the reduction in anxiety symptoms immediately post-intervention $(g=0.69)$ and 6 months post-intervention $(g=0.91)$, which is consistent with results from other online interventions for anxiety disorders ${ }^{59,60}$.

The finding that Ascend was associated with reductions in symptoms of anxiety as well as depression is unsurprising given that the intervention comprises evidence-based practices that have previously been shown to be effective at treating both conditions ${ }^{61}$. In addition, depression and anxiety are highly comorbid, suggesting the presence of shared underlying mechanisms that may respond to 
similar treatment methods ${ }^{62}$. Indeed, baseline scores suggest that a large proportion of participants were experiencing anxiety symptoms pre-intervention, despite being recruited on the basis of having symptoms of depression. However, the magnitude of symptom reduction associated with Ascend was slightly larger for depression than for anxiety, likely reflecting the fact that the intervention was designed to address depression as the primary condition. Whilst previous online psychological interventions have often separated the treatment of depression and anxiety symptoms, the present results highlight the potential utility of app- and online-based interventions that can reduce symptoms associated with both conditions concurrently.

An important but largely unanswered question is who is likely to benefit the most from app-based psychological interventions, and what factors are predictive of the degree to which individuals benefit over time. Our previous feasibility study suggested that a greater volume of app-based practice predicted the occurrence of fewer depressive symptoms 4-weeks after completing the Ascend intervention ${ }^{38}$. In the present study, the degree of engagement with Ascend did not impact depression or anxiety scores at 6 - or 12 -months post-intervention, though this analysis was likely underpowered due to a large proportion of missing data at these time-points. It's also likely that a portion of the variance in scores at these time-points would have been driven by factors not captured by our study. Further research is needed to understand the factors and components of the intervention that are predictive of long-term benefits.

\section{Limitations}

As with the former feasibility study ${ }^{38}$, the present study used a non-randomized, uncontrolled design, which precludes causal inferences regarding the intervention and patient-reported outcome changes. In addition, the effect sizes reported in the present study are likely an overestimate of the true treatment effect relative to an active control or treatment as usual.

Further, approximately one-third of participants were taking antidepressants during (and presumably after) the intervention, making it difficult to preclude the possibility that the reduction in depression symptoms was caused or maintained by antidepressants ${ }^{63}$, or by the tendency for a proportion of depressed patients to naturally recover over a 12 -month period ${ }^{64}$, and not by the current intervention. Future randomized controlled trials that compare Ascend to treatment as usual over an extended period are required to address these limitations. 
Further, although engagement with the intervention was high, approximately half of all participants did not complete questionnaires at 6 - and 12 -months post-intervention. Thus, our estimates of the percentage of participants that experienced clinically significant improvements in depression symptoms was based on those with complete data only. Further, participants with fewer symptoms of depression post-intervention may have been more likely to engage with questionnaires, biasing estimates of the long-term treatment effect. This was addressed by using a robust pattern-mixture model approach and applying the most conservative clinical assumptions about the treatment effect for participants who dropped out or who were lost to follow-up. Nevertheless, such assumptions are inherently unverifiable, and recent guidelines highlight the importance of minimizing the likelihood of incomplete outcome data 65 .

Whilst the present study suggests that the intervention is associated with reductions in depression and anxiety symptoms, it does not address the underlying mechanisms or mediators of outcome change. Since the intervention encompasses techniques and practices from multiple approaches (CBT, MM, and BAT), as well as remote therapist and peer-support, it is difficult to differentiate which components of the intervention are the most effective. Such information could assist with the design and optimization of future iterations of Ascend and other app-based interventions for mental health (see $\left.{ }^{66}\right)$.

Finally, the study included self-selected participants who may have shown a higher degree of motivation to engage with the intervention, and the majority of participants were female. Thus, the study sample is not representative of the wider population of individuals with elevated symptoms of depression and/or anxiety. Future studies should address this by using more robust recruitment strategies and more representative study samples.

\section{Conclusion}

Depression is a serious and growing problem that causes individual suffering, and huge economic and societal costs worldwide. Many individuals with depression are unable to access appropriate treatment, with high costs and a lack of trained professionals being major barriers. Scalable, lowcost, app-based interventions such as Ascend are designed to overcome these barriers and may help to significantly reduce the burden of anxiety and depression. Further research is needed to investigate the efficacy of Ascend in comparison to control groups and other established treatments for depression and/or anxiety. 


\section{Acknowledgements}

We wish to express our gratitude to all participants for their efforts, and to the staff and therapists at Meru Health who were involved in developing, implementing and supporting the study intervention.

\section{Author Contributions}

M.E., K.R., A.R., and V.F-H. conceived of the study and designed it. M.E. performed statistical analysis, interpreted the data, and drafted the manuscript. K.R., A.N., O.H., and A.R. oversaw the design and creation of the study intervention. A.N., and O.H. oversaw data collection and archiving. V. F-H. contributed to interpretation of the data. All authors revised the manuscript and approved the final content.

\section{Competing Interests Statement}

All authors are employed, receive a salary, and/or hold equity at Meru Health Inc. K.R. serves as the chief executive officer of Meru Health Inc. and owns a large share of the company's stocks. A.N. serves as the chief technology officer of Meru Health Inc. and owns a large share of the company's stocks.

\section{References}

1.

4.

5.
Mathers, C. D. \& Loncar, D. Projections of Global Mortality and Burden of Disease from 2002 to 2030. PLoS Medicine 3, e442 (2006).

Richards, D. Prevalence and clinical course of depression: a review. Clin Psychol Rev 31, 11171125 (2011).

Moussavi, S. et al. Depression, chronic diseases, and decrements in health: results from the World Health Surveys. Lancet 370, 851-858 (2007).

Lopez, A. D. \& Mathers, C. D. Measuring the global burden of disease and epidemiological transitions: 2002-2030. Ann Trop Med Parasitol 100, 481-499 (2006).

Papakostas, G. I. \& Ionescu, D. F. Updates and trends in the treatment of major depressive disorder. J Clin Psychiatry 75, 1419-1421 (2014). 

noncompliance: the potential benefits of online support. Int Clin Psychopharmacol 25, 288-296 (2010).

7.

8.

9.

10.

11.

12.

13.

14.

15.

16.

17.

18.

19.

20.

Cipriani, A. et al. Comparative efficacy and acceptability of 21 antidepressant drugs for the acute treatment of adults with major depressive disorder: a systematic review and network meta-analysis. Lancet 391, 1357-1366 (2018).

Cuijpers, P., van Straten, A., Andersson, G. \& van Oppen, P. Psychotherapy for Depression in Adults: A Meta-Analysis of Comparative Outcome Studies. FOCUS 8, 75-75 (2010).

Hofmann, S. G., Sawyer, A. T., Witt, A. A. \& Oh, D. The effect of mindfulness-based therapy on anxiety and depression: A meta-analytic review. J Consult Clin Psychol 78, 169-183 (2010). Substance Abuse and Mental Health Services Administration. Key substance use and mental health indicators in the United States: Results from the 2016 National Survey on Drug Use and Health (HHS Publication No. SMA 17-5044, NSDUH Series H-52). (Rockville, MD: Center for Behavioral Health Statistics and Quality, Substance Abuse and Mental Health Services Administration, 2017).

Kohn, R., Saxena, S., Levav, I. \& Saraceno, B. The treatment gap in mental health care. Bull. World Health Organ. 82, 858-866 (2004).

Mohr, D. C. et al. Perceived barriers to psychological treatments and their relationship to depression. Journal of Clinical Psychology 66, 394-409 (2010).

Weil, T. P. Insufficient dollars and qualified personnel to meet United States mental health needs. J. Nerv. Ment. Dis. 203, 233-240 (2015).

Souery, D. et al. Clinical factors associated with treatment resistance in major depressive disorder: results from a European multicenter study. J Clin Psychiatry 68, 1062-1070 (2007). Rush, A. J. et al. Acute and longer-term outcomes in depressed outpatients requiring one or several treatment steps: a STAR*D report. Am J Psychiatry 163, 1905-1917 (2006).

$\mathrm{Hu}, \mathrm{X}$. H. et al. Incidence and duration of side effects and those rated as bothersome with selective serotonin reuptake inhibitor treatment for depression: patient report versus physician estimate. J Clin Psychiatry 65, 959-965 (2004).

Cartwright, C., Gibson, K., Read, J., Cowan, O. \& Dehar, T. Long-term antidepressant use: patient perspectives of benefits and adverse effects. Patient Preference and Adherence Volume 10, 1401-1407 (2016).

Pigott, H. E. STAR*D: A Tale and Trail of Bias. Ethical Human Psychology and Psychiatry 13, 6-28 (2011).

Coplan, J. D., Aaronson, C. J., Panthangi, V. \& Kim, Y. Treating comorbid anxiety and depression: Psychosocial and pharmacological approaches. World J Psychiatry 5, 366-378 (2015).

Mohr, D. C. et al. Barriers to psychotherapy among depressed and nondepressed primary care patients. Ann Behav Med 32, 254-258 (2006). 
Weddington, W. W. Adherence by medical-surgical inpatients to recommendations for outpatient psychiatric treatment. Psychother Psychosom 39, 225-235 (1983).

Strecher, V. Internet methods for delivering behavioral and health-related interventions (eHealth). Annu Rev Clin Psychol 3, 53-76 (2007).

Wilhelmsen, M. et al. Motivation to persist with internet-based cognitive behavioural treatment using blended care: a qualitative study. BMC Psychiatry 13, 296 (2013).

Spek, V. et al. Internet-based cognitive behaviour therapy for symptoms of depression and anxiety: a meta-analysis. Psychological Medicine 37, 319 (2006).

Richards, D. \& Richardson, T. Computer-based psychological treatments for depression: A systematic review and meta-analysis. Clin Psychol Rev 32, 329-342 (2012).

Andersson, G. \& Cuijpers, P. Internet-Based and Other Computerized Psychological Treatments for Adult Depression: A Meta-Analysis. Cogn Behav Ther 38, 196-205 (2009).

Ahern, E., Kinsella, S. \& Semkovska, M. Clinical efficacy and economic evaluation of online cognitive behavioral therapy for major depressive disorder: a systematic review and metaanalysis. Expert Review of Pharmacoeconomics \& Outcomes Research 18, 25-41 (2017). Spijkerman, M. P. J., Pots, W. T. M. \& Bohlmeijer, E. T. Effectiveness of online mindfulnessbased interventions in improving mental health: A review and meta-analysis of randomised controlled trials. Clin Psychol Rev 45, 102-114 (2016).

Karyotaki, E. et al. Efficacy of Self-guided Internet-Based Cognitive Behavioral Therapy in the Treatment of Depressive Symptoms: A Meta-analysis of Individual Participant Data. JAMA Psychiatry 74, 351-359 (2017).

Carlbring, P., Andersson, G., Cuijpers, P., Riper, H. \& Hedman-Lagerlöf, E. Internet-based vs. face-to-face cognitive behavior therapy for psychiatric and somatic disorders: an updated systematic review and meta-analysis. Cogn Behav Ther 47, 1-18 (2018).

Poushter, J. Smartphone ownership and internet usage continues to climb in emerging economies. Pew Research Center (2016). Available at: (Accessed: 14 January 2019)

Proudfoot, J. et al. Community attitudes to the appropriation of mobile phones for monitoring and managing depression, anxiety, and stress. J. Med. Internet Res. 12, e64 (2010). Torous, J. et al. Patient Smartphone Ownership and Interest in Mobile Apps to Monitor Symptoms of Mental Health Conditions: A Survey in Four Geographically Distinct Psychiatric Clinics. JMIR Ment Health 1, e5 (2014).

Donker, T. et al. Smartphones for smarter delivery of mental health programs: a systematic review. J. Med. Internet Res. 15, e247 (2013).

Firth, J. et al. The efficacy of smartphone-based mental health interventions for depressive symptoms: a meta-analysis of randomized controlled trials. World Psychiatry 16, 287-298 (2017).

Firth, J. et al. Can smartphone mental health interventions reduce symptoms of anxiety? A meta-analysis of randomized controlled trials. Journal of Affective Disorders 218, 15-22 (2017). 
37.

38.

39.

40.

41.

42.

43.

44.

45.

46.

47.

48.

49.

50.

51.

52.

53.

54.

55.

Huguet, A. et al. A Systematic Review of Cognitive Behavioral Therapy and Behavioral Activation Apps for Depression. PLoS ONE 11, e0154248 (2016).

Goldin, P. R. et al. Feasibility of a Therapist-Supported, Mobile Phone-Delivered Online Intervention for Depression: Longitudinal Observational Study. JMIR Formative Research 3, e11509 (2019).

Kabat-Zinn, J. Wherever You Go, There You Are: Mindfulness meditation for everyday life. (Hachette Books, 1994).

Morgan, D. Mindfulness-Based Cognitive Therapy for Depression: A New Approach to Preventing Relapse. Psychotherapy Research 13, 123-125 (2003).

Beck, A. T. Cognitive Therapy and the Emotional Disorders. (Penguin, 1979).

Jacobson, N. S., Martell, C. R. \& Dimidjian, S. Behavioral Activation Treatment for Depression: Returning to Contextual Roots. Clinical Psychology: Science and Practice 8, 255-270 (2001). Kroenke, K., Spitzer, R. L. \& Williams, J. B. The PHQ-9: validity of a brief depression severity measure. J Gen Intern Med 16, 606-613 (2001).

Arroll, B. et al. Validation of PHQ-2 and PHQ-9 to screen for major depression in the primary care population. Ann Fam Med 8, 348-353 (2010).

McMillan, D., Gilbody, S. \& Richards, D. Defining successful treatment outcome in depression using the PHQ-9: A comparison of methods. Journal of Affective Disorders 127, 122-129 (2010). Löwe, B., Kroenke, K., Herzog, W. \& Gräfe, K. Measuring depression outcome with a brief selfreport instrument: sensitivity to change of the Patient Health Questionnaire (PHQ-9). Journal of Affective Disorders 81, 61-66 (2004).

Spitzer, R. L., Kroenke, K., Williams, J. B. W. \& Löwe, B. A brief measure for assessing generalized anxiety disorder: the GAD-7. Arch. Intern. Med. 166, 1092-1097 (2006).

Löwe, B. et al. Validation and Standardization of the Generalized Anxiety Disorder Screener (GAD-7) in the General Population. Med Care 46, 266-274 (2008).

Kroenke, K. et al. Patient Health Questionnaire Anxiety and Depression Scale: Initial Validation in Three Clinical Trials. Psychosomatic Medicine 78, 716-727 (2016).

Bates, D., Mächler, M., Bolker, B. \& Walker, S. Fitting Linear Mixed-Effects Models Using Ime4. Journal of Statistical Software 67, 1-48 (2015).

Hothorn, T., Bretz, F. \& Westfall, P. Simultaneous inference in general parametric models. Biom J 50, 346-363 (2008).

Baraldi, A. N. \& Enders, C. K. An introduction to modern missing data analyses. J Sch Psychol 48, 5-37 (2010).

Ratitch, B., O'Kelly, M. \& Tosiello, R. Missing data in clinical trials: from clinical assumptions to statistical analysis using pattern mixture models. Pharm Stat 12, 337-347 (2013).

Fox, J. \& Weisberg, S. An R Companion to Applied Regression. (SAGE Publications, 2018).

Ratitch, B. \& OKelly, M. Pattern-Mixture Models as Linear Combinations of Least Squares Means from MMRM with Delta Method Variance Estimation. PharmaSUG (2012). 
56. Schlosser, D. A. et al. The feasibility, acceptability, and outcomes of PRIME-D: A novel mobile intervention treatment for depression. Depression and Anxiety 34, 546-554 (2017).

57. Meyer, B. et al. Effects of an Internet intervention (Deprexis) on severe depression symptoms: Randomized controlled trial. Internet Interventions 2, 48-59 (2015).

58. Watts, S. et al. CBT for depression: a pilot RCT comparing mobile phone vs. computer. BMC Psychiatry 13, 808 (2013).

59. Hofmann, S. G. \& Smits, J. A. J. Cognitive-behavioral therapy for adult anxiety disorders: a metaanalysis of randomized placebo-controlled trials. J Clin Psychiatry 69, 621-632 (2008).

60. Reger, M. A. \& Gahm, G. A. A meta-analysis of the effects of internet- and computer-based cognitive-behavioral treatments for anxiety. Journal of Clinical Psychology 65, 53-75 (2009).

61. Saddichha, S., Al-Desouki, M., Lamia, A., Linden, I. A. \& Krausz, M. Online interventions for depression and anxiety - a systematic review. Health Psychol Behav Med 2, 841-881 (2014).

62. Zbozinek, T. D. et al. Diagnostic overlap of generalized anxiety disorder and major depressive disorder in a primary care sample. Depression and Anxiety 29, 1065-1071 (2012).

63. Simon, G. E. Long-term prognosis of depression in primary care. Bull. World Health Organ. 78, 439-445 (2000).

64. Spijker, J. et al. Duration of major depressive episodes in the general population: Results from the Netherlands Mental Health Survey and Incidence Study (NEMESIS). Br J Psychiatry 181, 208213 (2002).

65. Little, R. J. et al. The prevention and treatment of missing data in clinical trials. N. Engl. J. Med. 367, 1355-1360 (2012).

66. Cuijpers, P., Cristea, I. A., Karyotaki, E., Reijnders, M. \& Hollon, S. D. Component studies of psychological treatments of adult depression: A systematic review and meta-analysis.

Psychother Res 29, 15-29 (2019). 